\title{
Current Use of Steroids in Critical Care
}

\author{
Anahita Dabo Trubelja* \\ Department of Anesthesiology and Critical Care Medicine, Memorial Sloan-Kettering Cancer Center, USA
}

Received: December 11, 2013; Accepted: March 10, 2014, Published: March 12, 2014

*Corresponding author: Anahita Dabo Trubelja, Department of Anesthesiology and Critical Care Medicine, Memorial Sloan-Kettering Cancer Center, 1275, York Avenue, New York, USA, Tel: 212-639-3279; Fax: 646-422-2088; E-mail: DaboA@mskcc.org

\begin{abstract}
Septic shock is characterized by an uncontrolled systemic inflammatory response that contributes to organ dysfunction, failure and eventually death. The importance of the adrenal glands for survival under conditions of physiologic stress has been known since the early 20th century. Clinical studies explored the potential therapeutic role of corticosteroids in the treatment of sepsis and septic shock. Despite controversies on the benefit-to-risk ratio, they are widely used. The longstanding adoption of corticosteroids in the treatment of severe sepsis likely relies on the prompt reversal of septic shock often seen at the bedside. This current review was designed to provide readers with a clear understanding and rationale for using corticosteroids, while presenting a review of the Surviving Sepsis Guidelines and the results from the implementation of the Surviving Sepsis Campaign.
\end{abstract}

Keywords: Sepsis; Septic Shock; Corticosteroids; Critical IllnessRelated Corticosteroid Insufficiency (CIRCI); Relative Adrenal Insufficiency (RAI); Surviving Sepsis Guidelines; Surviving Sepsis Campaign

The hypothalamic-pituitary-adrenal (HPA) integrity is a major factor of the host's response to stress. During sepsis the HPA axis is activated and the ACTH that is released from the pituitary gland enhances adrenal activity, resulting in high plasma cortisol level. This state of continuous adrenal secretory activity leads to relative adrenal insufficiency (RAI). In view of the complexity of the stress response, the level of plasma cortisol present in the body is often insufficient to meet the demands of the inflammatory response and the resulting cortisol levels may be high, normal or low. This state is also known as critical illness-related corticosteroid insufficiency (CIRCI). Effects of CIRCI include alteration in systemic inflammatory response and altered cardiovascular function. Cortisol plays an important role in controlling vascular tone as it increases sensitivity to vasopressors. There are also a number of neuro-peptides which act on the HPA integrity. The most commonly known is vasopressin, which has shown to increase endogenous adrenal ACTH secretion. Apelin and co-peptin are two other neuropeptides that act on the pre-vasopressin molecule. They decrease the production of vasopressin, thereby contributing to the host's response to stress and may account for the variable plasma cortisol level [1-6].
The altered HPA leads to a biphasic pattern during critical illness uncoupling of ACTH and cortisol that may account for an alternative pathway not mediated by ACTH. Cortisol activates the steroid receptor complex at the cellular level of many different genes and inhibits the synthesis of mediators of inflammation such as cytokines, IL, cell adhesion molecules, TNF-a, and NF-kB. In critical illness these pathways are impaired. Some proposed mechanisms include decrease production at all levels of the HPA axis, dysfunction of the glucocorticoid receptors, and structural damage to the adrenal gland from hemorrhage or infarction. Failure of clinical improvement in sepsis has been associated with failure to activate the steroid complex receptors that down regulate the transcription of inflammatory cytokines. Systemic inflammation induces corticosteroid resistance or tissue resistance, despite adequate cytoplasmic and serum cortisol levels [1,7-10].

RAI is defined as having a baseline cortisol level $<20 \mathrm{cmg} / \mathrm{dL}$. Since baseline cortisol levels in patients with severe sepsis can be high, using an incremental change in cortisol level of $<9 \mathrm{cmg} /$ $\mathrm{dL}$ may be more useful in assessing baseline measurements. A corticotropin stimulation test is used to assess RAI by administering $250 \mathrm{cmg}$ of corticotropin hormone and obtaining a basal plasma cortisol level. Samples are then drawn 30 and 60 minutes after the given dose to measure plasma cortisol levels. A 3-level Prognostic Classification System is used to evaluate RAI:

a. Good: Cortisol concentrations before corticotropin test $\leq$ $34 \mathrm{mcg} / \mathrm{dL}$ and response to corticotropin test $>9 \mathrm{mcg} / \mathrm{dL}$

b. Intermediate: Cortisol concentrations before corticotropin test $\leq 34 \mathrm{mcg} / \mathrm{dL}$ and response to corticotropin test $\leq 9$ $\mathrm{mcg} / \mathrm{dL}$, or cortisol concentrations before corticotropin test $>34 \mathrm{mcg} / \mathrm{dL}$ and response to corticotropin test $>9$ $\mathrm{mcg} / \mathrm{dL}$

c. Poor: Cortisol concentrations before corticotropin test $>$ $34 \mathrm{mcg} / \mathrm{dL}$ and response to corticotropin test $\leq 9 \mathrm{mcg} / \mathrm{dL}$ [10].

Steroids have been studied for many decades. In the 1980's, some studies reported steroid replacement therapy as having a beneficial outcome in treating septic patients, particularly when routine high-dose methylprednisolone (up to $30 \mathrm{mg} / \mathrm{kg}$ ) was 
given. Although some of these studies showed promising results, duration and dose of corticosteroids in the treatment of sepsis and septic shock remained controversial $[11,12]$.

In the early 1990's, there was renewed interest to study the effect of a prolonged course of low-dose steroids, incidence of RAI, and factors associated with mortality. There was also a special interest in evaluating cortisol levels and cortisol response to the corticotropin test. Two studies by Ananne sought to answer these questions. The first study based the prognostic value of cortisol levels on a short corticotropin stimulation test in patients with septic shock. In this study patients were randomized within 8 hours of the onset of septic shock. Based on their study results a 3-Level Prognostic Classification System was created. The combination of basal cortisol levels (< or $>34 \mathrm{cmg} / \mathrm{dL}$ ) and the highest value of cortisol response to corticotropin $(<0 \mathrm{or}>9 \mathrm{cmg} /$ $\mathrm{dL}$ ) resulted in defining three different patterns of HPA axis activation of septic shock. These patterns were associated with three different outcomes:

1. $30 \%$ pts had adequate HPA axis activation with a basal cortisol level $<34$ and cortisol response $>9$. Lowest risk of death and median survival $>28$ days.

2. $20 \%$ pts had basal cortisol level $>34$ with occult adrenal insufficiency (cortisol response $<9$ ). Highest risk of death and median survival time of 5 days.

3. $50 \%$ pts had basal cortisol level $<34$ and cortisol response $<9$. Intermediate risk of death and median survival 12 days.

This study concluded that at the onset of septic shock basal plasma cortisol level and plasma response to corticotropin were independent predictors of 28 day mortality [10].

The second study addressed the use of a 7-day treatment course with low-dose hydrocortisone and fludrocortisone. The cortisol response was defined as the difference between the highest of concentrations taken before and after the test. In this study, RAI (i.e., non-responders) was defined by a response of $\leq 9 \mathrm{mcg} / \mathrm{dL}$. This trial found a significant reduction in the risk of death without an increase in adverse events in patients with septic shock and RAI. There were no differences in adverse events between the two groups [13].

In a multicenter, double-blind, placebo-controlled trial, the Corticus study randomized patients to hydrocortisone or placebo within 72 hours of the onset of septic shock. The Corticus study found that hydrocortisone treatment did not decrease mortality or time to shock reversal. In addition, hydrocortisone treatment was associated with an increased incidence of super infections, including new episodes of sepsis or septic shock. Adverse events such as hyperglycemia and hypernatremia were also noted. A difference in study design may explain the variation of study results between the two trials $[3,9,14]$.

In the Annane study patients with a primary source of infection in the lungs were enrolled within 8 hours of the onset of septic shock. Patients remained hypotensive despite fluid resuscitation and vasopressor therapy. In contrast, the Corticus study enrolled patients with a primary source of infection in the GI tract within 72 hours of the onset of septic shock. For these patients, septic shock was manifested by their hypotension or vasopressor requirement for at least one hour. This led to a disparity in the severity of illness between the two trials, with the Annane group having the sicker patients as measured by SAPS 2 scores and the incidence of mortality in the controlled group. These observations not only raised the issue of timing, but questioned whether sicker patients are more likely to benefit from overall steroid therapy and earlier administration of steroid therapy $[2,4,15,16]$.

In a published review of the risks and benefits of corticosteroid therapy, low-dose corticosteroid treatment ( $>5$ days) was favored. It also demonstrated a reduction in 28 day mortality and an overall reduction in hospital mortality. The length of stay for patients in the Intensive Care Unit (ICU) was shorter however there was no difference in the hospital length of stay. These patients also showed no evidence of an increased risk of gastrointestinal bleeding (GIB), super infections or neuromuscular weakness, however there was an increase in the incidence of hyperglycemia and hypernatremia $[1,17]$.

Another study addressing 3-day course versus 7-day course of low-dose hydrocortisone in patients with septic shock and relative adrenal insufficiency found no difference in mortality between the two groups with 28 day mortality as the primary endpoint showed [18].

Following these trials, a multi-disciplinary force developed a consensus statement on the diagnosis and management of corticosteroid insufficiency in critical illness. This statement was incorporated into the Surviving Sepsis Campaign Treatment Guidelines in 2008. It concluded that hydrocortisone may be given to adult septic shock patients who are fluid and vasopressor unresponsive after one hour, with the following recommendations:

1. An ACTH test is not necessary to identify subset of adults with septic shock who should receive hydrocortisone.

2. Dexamethasone should not be used.

3. Fludrocortisone may be given if hydrocortisone is not available.

4. Wean steroids when vasopressors are no longer required.

5. Doses $>300 \mathrm{mg} /$ day not be used.

6. Steroids should be not given for the treatment of sepsis in absence of septic shock [5,19-25].

Since its implementation, the effectiveness of the guidelines and treatment bundles has been evaluated in a prognostic manner. One study performed the two-part sepsis bundle in a community hospital and found a positive impact on clinical outcome in the number of days on vasopressor therapy, dialysis and mortality [26]. It is important to note that corticosteroid treatment is just one part of the Surviving Sepsis Campaign guidelines. Therefore, 
we cannot conclude that corticosteroid treatment alone is beneficial for survival, but rather the implementation of the whole package.

In 2012, the Surviving Sepsis Campaign published its results since the implementation of its guidelines. The adjusted hospital mortality was significantly higher in the group that received low-dose steroids for septic shock compared to those who did not. This increase in mortality was consistently higher even in patients who received corticosteroids within 8 hours of the onset of septic shock $[16,27,28]$. Reports from two large international sepsis registries, EDUSEPSIS and PROGRESS, have not supported the use of low-dose steroids in sepsis and septic shock [29]. Moreover, the Bayesian analysis, a statistical methodology used to solve clinical controversies, supports these findings but do not support the use of low-dose steroids in sepsis [30].

Varying practices on the use of low-dose corticosteroids in the treatment of septic shock remains. Many studies have reported a significant improvement in time to shock reversal after treatment with corticosteroids despite evidence showing no benefit in decrease mortality $[8,16,31]$. Several meta-analysis have failed to reproduce the positive results seen in previous studies. The use of corticosteroids in the treatment of sepsis and septic shock in the setting of RAI remains controversial [3,15,27,32]. Despite these observations the use of low-dose steroids continues to be incorporated in the management of sepsis and septic shock as corticosteroids have shown a faster time to shock reversal [8,3335]. These large sepsis registries provide a description of the management practices and outcomes based on the guidelines and are therefore secondary analysis $[27,31]$. The ambiguity remains. Large randomized controlled trials are needed to define the role of low-dose steroids, identify patients who would benefit and to define a recommended dose and duration in treating sepsis and septic shock. The Adrenal trial is underway and aims to address various prescribing practices of corticosteroids, as well as the outcome at 90 days, the patient's length of ICU stay and quality of life at 6 months [36]. The collaboration of new trials and the Surviving Sepsis Campaign will provide new clinical evidence to incorporate in current guidelines.

\section{References}

1. Cooper MS, Stewart PM (2003) Corticosteroid insufficiency in acutely ill patients. N Engl J Med 348(8): 727-734.

2. Marik PE (2009) Critical illness-related corticosteroid insufficiency. Chest 135(1): 181-193.

3. Annetta M, Maviglia R, Proietti R, Antonelli M (2009) Use of corticosteroids in critically ill septic patients: a review of mechanisms of adrenal insufficiency in sepsis and treatment. Curr Drug Targets 10(9): 887-894.

4. Maxime V, Lesur O, Annane D (2009) Adrenal insufficiency in septic shock. Clin Chest Med 30(1): 17-27.

5. Mesotten D, Vanhorebeek I, Van den Berghe G (2008) The altered adrenal axis and treatment with glucocorticoids during critical illness. Nat Clin Pract Endocrinol Metab 4(9): 496-505.

6. Peng J, Du B (2010) Sepsis-related stress response: known knowns, known unknowns, and unknown unknowns. Crit Care 14(4): 179.
7. Molenaar N, Bijkerk RM, Beishuizen A, Hempen CM, de Jong MF, et al. (2012) Steroidogenesis in the adrenal dysfunction of critical illness: impact of etomidate. Crit Care 16(4): R121.

8. Sprung CL, Annane D, Singer M, Moreno R, Keh D, et al. (2011) Glucocorticoids in sepsis: dissecting facts from fiction. Crit Care 15(5): 446.

9. Sprung CL, Goodman S, Weiss YG (2009) Steroid therapy of septic shock. Crit Care Clin 25(4): 825-834.

10.Annane D, Bellissant E, Sebille V, Lesieur O, Mathieu B, et al. (1988) Impaired pressor sensitivity to noradrenaline in septic shock patients with and without impaired adrenal function reserve. $\mathrm{Br} \mathrm{J}$ Clin pharmacol 46(6): 589-597.

11. Bone RC, Fisher CJ, Clemmer TP, Slotman GJ, Metz CA, et al. (1987) A controlled clinical trial of high-dose methylprednisolone in the treatment of severe sepsis and septic shock. N Engl J Med 317(11): 653-658.

12.(1987) Effect of high-dose glucocorticoid therapy on mortality in patients with clinical signs of systemic sepsis. The Veterans Administration Systemic Sepsis Cooperative Study Group. N Engl J Med 317(11): 659-665.

13. Annane D (2001) Corticosteroids for septic shock. Crit Care Med 29(7 Suppl): S117-120.

14. Toma A, Stone A, Green RS, Gray S (2011) Steroids for patients in septic shock: the results of the CORTICUS trial. CJEM 13(4): 273-276.

15. Annane D, Bellissant E, Bollaert PE, Briegel J, Confalonieri M, et al. (2009) Corticosteroids in the treatment of severe sepsis and septic shock in adults: a systematic review. JAMA 301(22): 2362-2375.

16. Casserly B, Gerlach H, Phillips GS, Lemeshow S, Marshall JC, et al. (2012) Low-dose steroids in adult septic shock: results of the Surviving Sepsis Campaign. Intensive Care Med 38(12): 1946-1954.

17. Balk RA (2003) Steroids for septic shock: back from the dead? (Pro). Chest 123(5 Suppl): 490S-499S.

18. Huh JW, Choi HS, Lim CM, Koh Y, Oh YM, et al. (2011) Low-dose hydrocortisone treatment for patients with septic shock: a pilot study comparing 3days with 7days. Respirology 16(7): 1088-1095.

19.Sprung CL, Annane D, Keh D, Moreno R, Singer M, et al. (2008) Hydrocortisone therapy for patients with septic shock. N Engl J Med 358(2): 111-124.

20. Ferrer R, Artigas A, Levy MM, Blanco J, Gonzalez DG, et al. (2008) Improvement in process of care and outcome after a multicenter severe sepsis educational program in Spain. JAMA 299(19): 22942303.

21. Dellinger RP, Levy MM, Carlet JM, Bion J, Parker MM, et al. (2008) Surviving Sepsis Campaign: international guidelines for management of severe sepsis and septic shock: 2008. Intensive care med 34(1): 1760.

22. Spoelstra-de Man AME, Girbes ARJ (2008) Comment on "Surviving Sepsis Campaign: International guidelines for management of severe sepsis and septic shock: 2008" by Dellinger et al. Intensive care medicine 34(6): 1160-1162.

23. Townsend SR, Schorr C, Levy MM, Dellinger RP (2008) Reducing mortality in severe sepsis: the Surviving Sepsis Campaign. Clin Chest Med 29(4): 721-733.

24.Perel A (2008) Bench-to-bedside review: the initial hemodynamic 
resuscitation of the septic patient according to Surviving Sepsis Campaign guidelines--does one size fit all?. Crit Care 12(5): 223.

25.Salluh JI, Bozza PT, Bozza FA (2008) Surviving sepsis campaign: a critical reappraisal. Shock 30 (Suppl 1): 70-72.

26. Patel GW, Roderman N, Gehring H, Saad J, Bartek W (2010) Assessing the effect of the Surviving Sepsis Campaign treatment guidelines on clinical outcomes in a community hospital. Ann pharmacother 44(11) 1733-1738.

27. Sherwin RL, Garcia AJ, Bilkovski R (2012) Do low-dose corticosteroids improve mortality or shock reversal in patients with septic shock? A systematic review and position statement prepared for the American Academy of Emergency Medicine. J Emerg Med 43(1): 7-12.

28. Patel GP, Balk RA (2012) Systemic steroids in severe sepsis and septic shock. Am J Respir Crit Care Med 185(2): 133-139.

29. Beale R, Janes JM, Brunkhorst FM, Dobb G, Levy MM, et al. (2010) Global utilization of low-dose corticosteroids in severe sepsis and septic shock: a report from the PROGRESS registry. Crit Care 14(3): R102.

30.Kalil AC, Sun J (2011) Low-dose steroids for septic shock and severe sepsis: the use of Bayesian statistics to resolve clinical trial controversies. Intensive Care Med 37(3): 420-429.

31. Dellinger RP, Levy MM, Rhodes A, Annane D, Gerlach H, et al. (2013) Surviving Sepsis Campaign: international guidelines for management of severe sepsis and septic shock, 2012. Intensive care medicine 39(2): 165-228.

32. Batzofin BM, Sprung CL, Weiss YG (2011) The use of steroids in the treatment of severe sepsis and septic shock. Best pract Res Clin Endocrinol Metab 25(5): 735-743.

33. Sprung CL, Brezis M, Goodman S, Weiss YG (2011) Corticosteroid therapy for patients in septic shock: some progress in a difficult decision. Crit Care Med 39(3): 571-574.

34.Sprung CL, Goodman S, Weiss YG (2011) Steroid therapy of septic shock. Crit Care Nurs Clin North Am 23(1): 171-180.

35. Sprung CL, Batzofin B, Goodman S, Weiss Y (2011) Steroid treatment for patients with severe sepsis and septic shock. Intensive care medicine 37(9): 1566.

36. Venkatesh B, Myburgh J, Finfer S, Webb SA, Cohen J, et al. (2013) The ADRENAL study protocol: adjunctive corticosteroid treatment in critically ill patients with septic shock. Crit Care Resusc 15(2): 83-88. 